\title{
Kinesthetic Cues that Lead the Way
}

\author{
Tomohiro Amemiya \\ NTT Communication Science Laboratories \\ Japan
}

\section{Introduction}

Wayfinding is of vital importance to pedestrians walking in unfamiliar areas. Generally, pedestrians rely on directional information, street names, and landmarks [Bradley \& Dunlop, 2005]. Recently, many mobile devices, such as mobile smart phones, can provide us with detailed digital maps, global positioning information, and navigational information. These location-based data and services are usually presented on visual displays. However, the visual displays in mobile devices are very small, which makes it hard to see and use the data. With the increasing complexity of information, and the variety of contexts of its use, it becomes important to consider how other non-visual sensory channels, such as audition and touch, can be used to communicate necessary and timely information to users. Additionally, there are a number of user groups, such as visually impaired people and the emergency services, who also require non-visual access to geographical data.

Kinesthetic stimulation, such as that for pulling or pushing the hand, has the potential to be more intuitive and expressive than cutaneous stimulation, such as rumbling vibration, in conveying direction information because force feedback devices can indicate a onedimension direction directly. Although a substantial number of force feedback devices have been developed in the last twenty years, most of them use either mechanical linkage to establish a fulcrum relative to the ground (Massie \& Salisbury, 1994), use a huge air compressor (Suzuki et al., 2002; Gurocak et al., 2003), or require wearing a heavy device (Hirose et al., 2001). Physical constraints mean that none of them can be used in portable information devices. Some portable "torque" displays have been proposed, based on the gyro effect (Yano et al., 2003) or angular momentum change (Tanaka et al., 2001) have been proposed; however, they can produce neither a constant force nor a translational force without also producing a reaction force; they can generate only a transient rotational force since they use a change in angular momentum. Recently, there have been a number of proposals for generating both constant and directional forces without an external fulcrum by using two oblique motors whose velocity and phase are controlled (Nakamura \& Fukui, 2007), by shifting the center-of-mass of a device dynamically to simulate kinesthetic inertia (Swindells et al., 2003), and by producing an air pressure field with airborne ultrasound (Iwamoto et al., 2008).

In contrast, our idea is to exploit the characteristics of human perception to devise a new force perception method for portable information devices that can generate a translation force sensation with a long duration (Amemiya et al. 2005; Amemiya and Maeda 2009). The method uses an asymmetric oscillation, where brief intense pulses of acceleration alternate 
with longer periods of low-amplitude recovery. Although the net acceleration is zero, humans perceive a sustained force sensation in the direction of the pulses. This is attributed to the nonlinear relationship between perceived acceleration and physical acceleration. We built a handheld prototype that generates periodic motion through asymmetric acceleration, in which asymmetric oscillation is generated by a swinging slider-crank mechanism. Our previous findings indicated that the pulse frequency determines the effective generation of the kinesthetic illusion of being pulled. In this chapter, we present a new hybrid configuration comprising a swinging slider-crank mechanism and a cam mechanism as an approach to fabricating a smaller force feedback system for portable information devices and describe an experiment in which we conducted an empirically examined turn-by-turn navigation with the device used by pedestrians with visual impairments. The results show the device intuitively conveys turning instructions and has potential to be used by untrained users.

\section{Pseudo-attraction force}

\subsection{Haptic sensory illusion}

The study of illusions can provide valuable insights into not only human perceptual mechanisms but also the design of new human interfaces. To generate a sustained translational force without grounding, we focused on the characteristics of human perception, which until now have been neglected or inadequately implemented in haptic devices. Although we human beings always interact with the world through human sensors and effectors, the perceived world is not identical to the physical world. For instance, different spectra can elicit the same color in human perception. When we watch television, the images on TV (a combination of RGB colors) we see are different from what we see through windows, i.e., a natural image is a composition of all wavelengths of light. Furthermore, animation actually consists of a series of still pictures in a flip book. Different stimuli can produce almost the same percept, which, though it may seem strange, is normal for humans. Since some illusions are very stable independent of individual variation, we can apply those illusions in practice, such as in designing human interfaces, if we can figure out ways to convert them to subjectively equivalent percepts. Hayward has pointed out that illusions are at the basis of virtually all technological displays (Hayward 2008), mentioning this also includes haptic interfaces.

\subsection{Principle}

The kinaesthetic illusion of being pulled or pushed, discovered by the authors (Amemiya et al. 2005), can be used to design haptic interfaces. Using different acceleration patterns for two directions to create a perceived force imbalance, the method exploits the characteristics of human perception to generate a force sensation and thereby produce the sensation of directional pushing or pulling. Specifically, a quicker acceleration (stronger force) is generated for a very brief time in the desired direction, while a slower acceleration (weaker force) is generated over a longer period in the opposite direction. The internal human haptic sensors do not detect the slower acceleration (weaker force), so the original position of the mass is washed out. The result is that the user is tricked into perceiving a unidirectional force. This force sensation can be made continuous by repeating the motions. If the acceleration patterns are well considered and designed, a kinesthetic illusion of being pulled can be created because of this nonlinearity. 


\subsection{Requirements}

There are still many aspects of the manifestation of the kinaesthetic illusion of the pseudoattraction force that are not well understood, but putative mechanisms have been accumulating. We know that no directional force is felt if the mass is merely moved back and forth, but that using different acceleration patterns for the two directions to create a perceived force imbalance produces the perception of a pseudo-attraction force (Amemiya \& Maeda, 2009). The frequency of the oscillation plays an important role in eliciting the perception of a pseudo-attraction force. Oscillations with high frequency might create a continuous force sensation, but previous experimental results have shown that the performance decreases steadily at frequencies over ten cycles per second (Amemiya et al., 2008). In contrast, oscillations with low frequency tend to be perceived as a discrete knocked sensation. If we wish to create a sustained rather than a discrete force sensation, such as the sensation of being pulled continuously, the frequency should be in the five to ten cycles per second range. In addition, changes in the gross weight and the weight of the reciprocating mass affects the perceived force sensation. The threshold of the ratio of the gross weight and the weight of the reciprocating mass is $16 \%$, which is a rough standard for effective force perception in the developed prototype (Amemiya \& Maeda, 2009).

\section{Hardware design}

Our first prototype used a swinging-block slider-crank mechanism to create an asymmetric oscillation [Fig. 1(a)]. In the mechanism, a circular motion of constant speed (crank OB) is transformed into a curvilinear motion since a swinging linkage $\mathrm{BC}$ slides and turns around point $A$. The end point on the curvilinear motion (point $C$ ) is connected with a rod (point $D$ ), which slides along a linear slider with asymmetric acceleration back-and-forth. Because of the length of the linkages, especially linkage $\mathrm{CD}$ (rod), the overall length of the mechanism tends to be large at about $175 \mathrm{~mm}$ (Amemiya and Maeda 2008; 2009). Figure 1(b) shows the new mechanism, which is the equivalent mechanism of the previous one but with the length of linkage CD decreased to virtually zero. As in the previous prototype, a circular motion of constant speed (crank OB) is transformed into a curvilinear motion by a swinging-block slider-crank mechanism. The difference is that the end point on the curvilinear motion (point C) slides along a grooved cam, whose shape is a circular arc with a radius of CD, with point $\mathrm{D}$ as the center of the arc. This produces a reciprocating motion with asymmetric oscillation.

The mechanisms in Fig. 1 have a single DOF (degree of freedom). We previously developed a prototype of a two-dimensional force display by having one module based on the slidercrank mechanism mounted on a turntable. The direction of the force display module was set by driving the turntable with a belt drive system. Turntable rotation, however, took considerable time, which meant that immediacy was lost. To overcome the problem of turntable rotation, we adopted the summation of linearly independent force vectors. We then fabricated a new 2-DOF prototype to generate a force sensation in at least eight cardinal directions by the summation of linearly independent force vectors. To create the force display, we stacked four layers, each containing a single module and two of which were orthogonal. By combining the force vectors generated by each module, the force display can create a force sensation on a two-dimensional plane more quickly than the turntable approach. The force display has the potential to create a force sensation in any arbitrary direction on a two-dimensional plane if the amplitude of the force vector can be changed. The asymmetric oscillation $(\mathrm{F}(t))$ is given by 

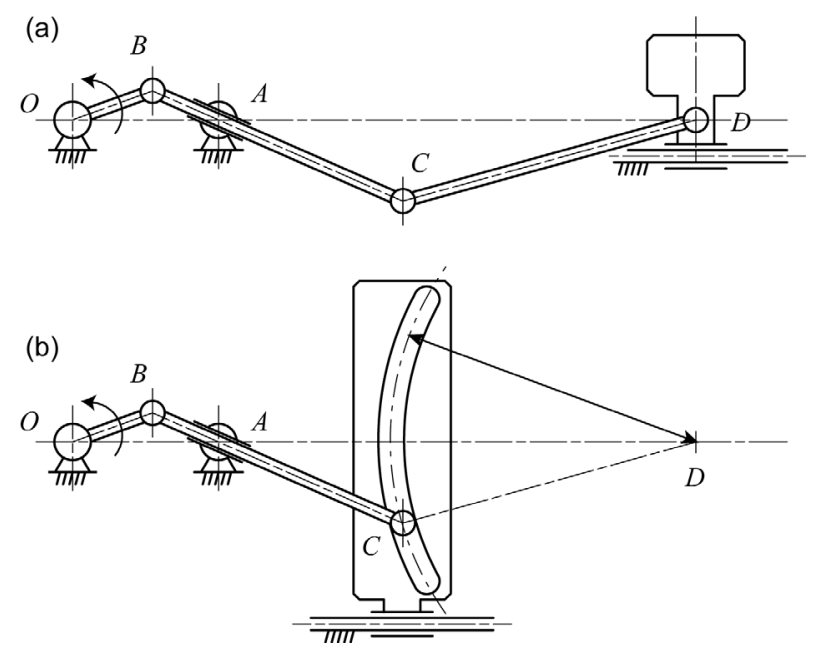

Fig. 1. Mechanisms for generating asymmetric oscillation in first prototype (a) and new one (b). The two mechanisms move identically

$$
F(t)=\sum_{j=1}^{n} m_{j} \frac{d^{2} x_{j}(t)}{d t^{2}} e_{j}
$$

where $m_{j}$ is the weight in module $j, n$ is the number of modules, and $d^{2} x_{j} / d t^{2}$ is the acceleration generated by module $j$. The acceleration $d^{2} x_{j} / d t^{2}$ is given by the second derivative with respect to time of the motion of the weight $x_{j}$. The equation for the motion of the weight in module $j$ is

$$
x_{j}(t)=l_{1} \cos \omega_{j} t+\mu_{j}\left(d-l_{1} \cos \omega_{j} t\right)+\sqrt{l_{3}-\left\{l_{1}\left(\mu_{j}-1\right) \sin \omega_{j} t\right\}^{2}}
$$

where

$$
\mu_{j}=\frac{l_{2}}{\sqrt{l_{1}^{2}+d^{2}-2 l_{1} d \cos \omega_{j} t}}
$$

and $x_{j}(t)=\mathrm{OD}, d=\mathrm{OA}, l_{1}=\mathrm{OB}, l_{2}=\mathrm{BC}, l_{3}=\mathrm{CD}$, and $\boldsymbol{\omega}_{j} t=\mathrm{AOB}$ in Fig. 1 . The $\boldsymbol{\omega}_{j}$ is the constant angular velocity, and $t$ is time. In the prototype, $d=28 \mathrm{~mm}, l_{1}=15 \mathrm{~mm}, l_{2}=60 \mathrm{~mm}$, $l_{3}=70 \mathrm{~mm}$, and $n=4$, and the unit vectors are $<\mathrm{e}_{j}, \mathrm{e}_{j+1}>=0,|| \mathrm{e}_{j}||=1$. All $m_{j}$ and $\omega_{j}$ values are identical, with $m_{j}=40 \mathrm{~g}, \boldsymbol{\omega}_{j} / 2 \pi=5 \mathrm{~Hz}$.

In the developed 2-DOF prototype, the output shaft of each motor (DC $6.0 \mathrm{~V}, 2232 \mathrm{R} 006 \mathrm{~S}$; Faulhaber) is mounted in a roller made of nylon. The roller drives the crank wheel by friction. The external diameter of the motor roller is $4 \mathrm{~mm}$, and that of crank wheel is $44 \mathrm{~mm}$. The reduction ratio is basically 1:11, but it changes slightly as a result of changes in factors such as temperature or pressure. The prototype weighs approximately $430 \mathrm{~g}$. The diameter of the base is the same as that of a compact disc (i.e., $120 \mathrm{~mm}$ ). The prototype is $36-\mathrm{mm}$ thick. Each weight on the slider is equipped with a photo-interrupter (PM-R24; SUNX Ltd.) to detect its position. The speed of each crank is stabilized at five counts per second by closed 
feedback loop control ( $\mathrm{P}$ control) with a microprocessor so that the signal intervals of the photo-interrupters are close to $200 \mathrm{~ms}$. When combining two orthogonal force vectors, the phases of the cranks are synchronized by another closed feedback loop (PID control) so that the onset intervals of the photo-interrupters are close to zero.

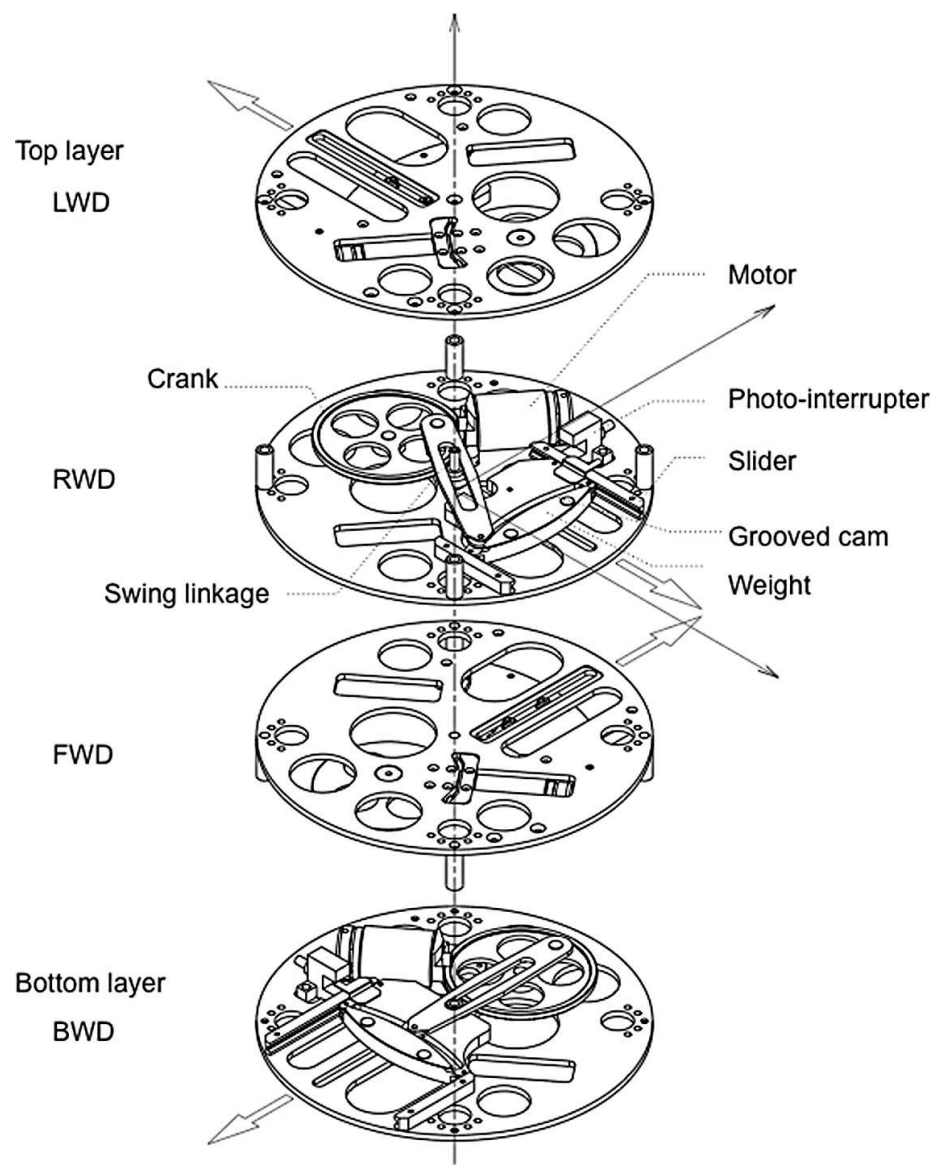

Fig. 2. Structure of the new prototype for generating pseudo-attraction force on a twodimensional plane

\section{Hardware evaluation}

Figure 3 shows the measured acceleration profile generated by the device at five counts per second. Acceleration values were calculated from the position data of each weight, which were acquired with a laser sensor (Keyence Inc., LK-G150, $10 \mathrm{kHz}$ sampling) with the bottom of the device fixed to a base. The acceleration profile of the top layer differed slightly from the others, due its distance from the fixed base. The effect of oscillation was augmented by the principle of leverage, leading to some degree of measurement error. The acceleration amplitude reached about $50 \%$ of the theoretical acceleration peak. We assume that the 
friction drive transmitted less torque than the previous gear drive. This clarified that there is a trade-off between the torque transmission efficiency and noise level when we select the friction drive or the gear drive.

Figure 4 shows examples of the response profiles of phase synchronization. The onset intervals between pairs consisting of two orthogonal modules were acquired from the photo-interrupters when the bottom of the device was fixed to the base. An onset interval of zero means that the two orthogonal modules are synchronized. The phases were synchronized within five seconds, which showed that the force display created a force sensation in the eight cardinal directions on a two-dimensional plane.

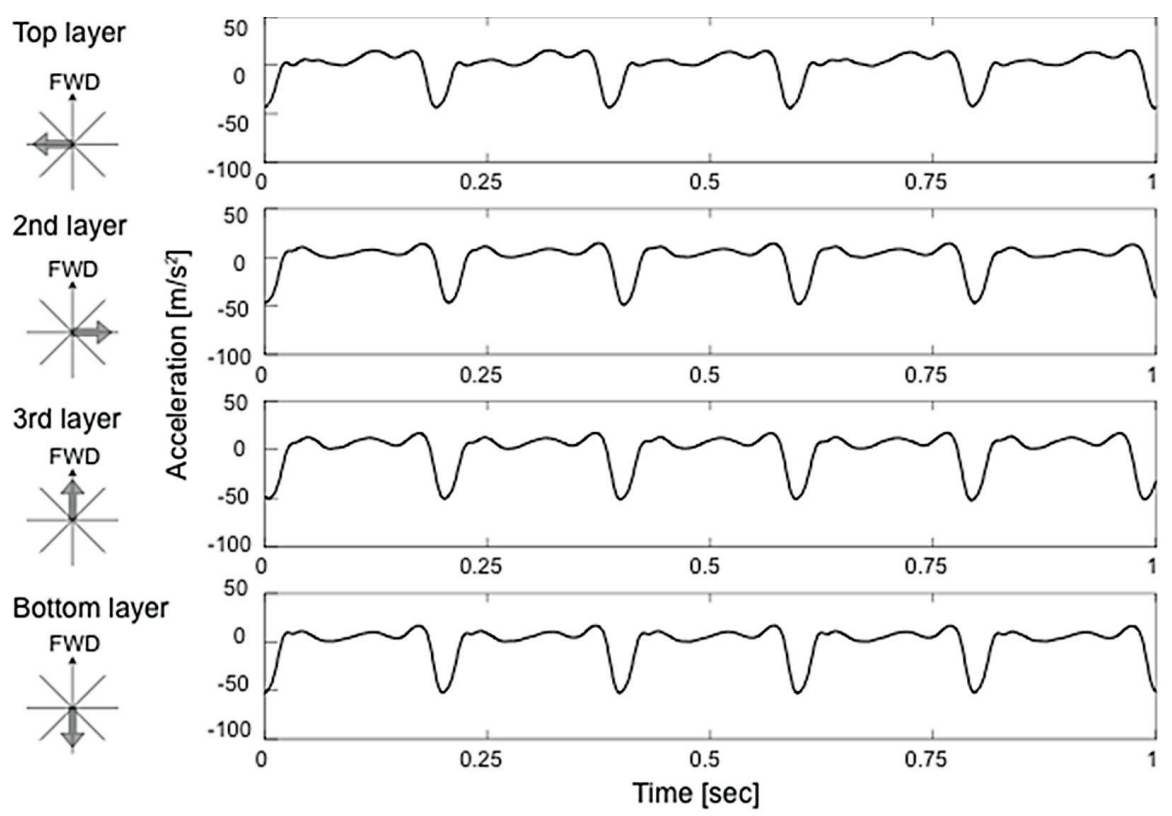

Fig. 3. Acceleration profile calculated from the position data measured with a laser sensor. The data were processed by a seventh order LPF Butterworth filter with a cut-off frequency of $100 \mathrm{~Hz}$

To drive the crank, the first prototype used a pinion gear and crown gears, whose axes were relatively displaced. The relative displacement of the gear axes caused gear noise, which annoyed the users. In fact, many people, including people who are blind, who have held the previous prototype, have complained about the noise. The sound pressure level of the noise generated by the previous prototype was measured in an anechoic room at NTT Communication Science Laboratories. A sound level meter (Rion, Inc., NL-31 Class 1) was used to measure the noise with the A-weighted sound pressure level (SPL). The sound level meter was fixed to a tripod at a height of $1.0 \mathrm{~m}$ from the ground and $30 \mathrm{~cm}$ from the prototype. The measured noise SPL showed that with the gear drive in the prior prototype, the noise level exceeded $60 \mathrm{~dB}(\mathrm{~A})$, whereas environmental noise level was $15 \mathrm{~dB}(\mathrm{~A})$. In contrast, the new prototype uses friction drive, and its noise level at frequencies of $3,5,10$ counts per second does not exceed $50 \mathrm{~dB}(\mathrm{~A})$, which shows that the friction drive emits much less noise. 

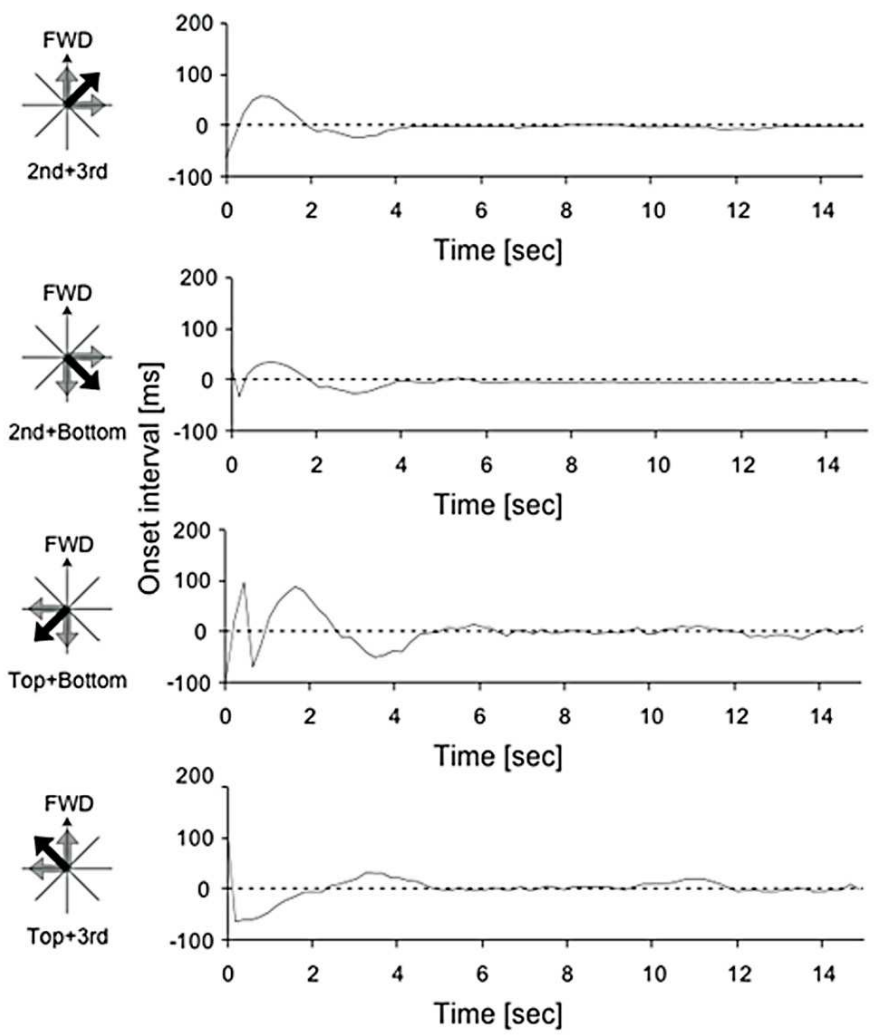

Fig. 4. Examples of measured phase synchronization responses for combinations of each module. The onset intervals obtained by the photo-interrupters were controlled to be close to zero by changing the angular velocity of the motor in the force display

\section{User studies}

We performed two user studies to investigate the applicability of the force display to pedestrian navigation. One was a psychophysical pilot study, which revealed that people who held the force display clearly sensed a directed force. The other was a navigation experiment related to predefined route guidance, in which people with visual impairments used the force display.

\subsection{Directional perception}

Five people without visual impairments (three right-handed men and two right-handed women, aged 22-34 years, 25.4 \pm 5.3 ), who were paid volunteers, participated in the psychophysical pilot study. None of the participants had any previous experience with force display prototypes. They were required to reply with one of eight cardinal directions after a five-second oscillating stimulus (north was defined as 0 degrees and the forward direction, and east as 90 degrees and right) without any prior training. Each participant experienced eight conditions $\times$ five trials, for a total of 40 trials (randomized). Visual and auditory effects 
were suppressed by having the participants wear eye-masks and earmuffs. The results showed that the force display provided a well-perceived directed force sensation (pseudoattraction force sensation) even though the measured acceleration peak was only half the expected value, and all responses from participants were almost identical to the direction of stimuli.

\subsection{Pedestrian navigation}

For the empirical experiment (Fig. 5), to verify the navigation system, we recruited people with visual impairments who were untrained. We measured the time required to complete a walking task, the level of ease of use and, the users' expectations of the guidance on a subjective scale, and collected user feedback. We built a human-size maze in the gymnasium of the Kyoto Prefectural School for the Visually Impaired, Japan. Since the streets and avenues in Kyoto are mainly laid out in a grid, the routes of the maze were designed in a checkerboard shaped.

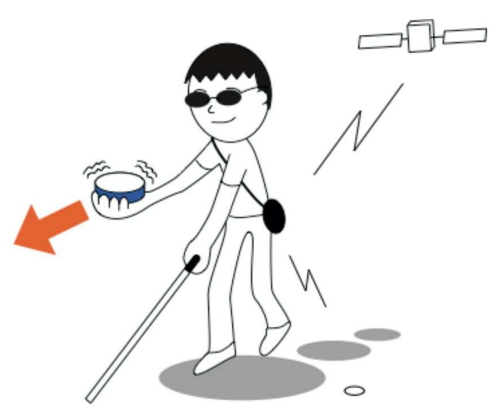

Fig. 5. Overview of kinesthetic navigation for pedestrians with visual impairments. After location data received from a satellite system or embedded sensors and orientation data had been collected and processed, a haptic cue of direction was presented to the pedestrian

\subsubsection{Method}

There were twenty-three visually impaired participants, 19 males and 4 females, who were volunteers from the Kyoto Prefectural School for the Visually Impaired. Ages ranged from 17 to 62 years $(30.0 \pm 14.7)$. Thirteen are totally blind, and the other ten are partially sighted. The participants all reported that they had no irregularities with their hands in terms of tactile perception at the time of the experiment, and they were all untrained. The research protocol was approved by local ethics committees. All participants provided written informed consent prior to testing. No participants underwent any prior training. Participants who usually used a cane when walking held a cane with one hand and the haptic direction indicator with the other. Others held the haptic direction indicator with the dominant hand. They carried a small shoulder bag (less than $600 \mathrm{~g}$ ), which contained a notebook computer (OQO model 02; OQO Inc.), a custom-build circuit with a microprocessor (PIC18F252; Microchip Tech. Inc.), and a 12-V battery (ENAX Corp.). Bluetooth 2.1 allowed the portable computer to communicate with the remote computer (ThinkPad X60s; Lenovo Corp.) within 100 meters. Since the global positioning system (GPS) did not work inside the gym with satisfactory accuracy, we used nine infrared sensors installed at the junctions of the maze as a local positioning system; they were connected to 
the remote computer. To produce white noise, a clip-shaped music player (iPod shuffle 2nd generation; Apple Inc.) and noise-cancelling headphones (Quiet Comfort 3; Bose Corp.) were used. To measure the yaw angle of the force display, a motion sensor (MDP-A3U9S; NEC TOKIN Corp.) was attached to it. The human-sized experimental labyrinth was formed with a series of foam panels $(1,800 \mathrm{~mm} \times 900 \mathrm{~mm})$ occupying a space of $9 \mathrm{~m} \times 15 \mathrm{~m}$. The pads were soft enough not to cause injury in collisions. The route and journey duration were automatically logged by the system with infrared sensors placed at the corners of the walls, captured by digital video cameras from the second floor (about $4 \mathrm{~m}$ from the ground), and manually noted by the experimenters.

A participant holding the haptic directional indicator was brought to one of three different departure points in the labyrinth. An experimenter walked behind the participant to ensure his/her safety. First, the force display presented a sensation of pushing forward. The participant then started walking from the departure point. The participant was guided by the force display to turn left or right at a certain turning point, and there were totally nine possible turning points. At that point, the infrared sensor detected the arrival of the participant. In response, the remote computer connected to the infrared sensors sent the turn instruction to the notebook computer in the participant's bag. The direction of the force vector (go straight; turn left or right) was initially determined by the predefined route at each turning point and automatically updated to one of the eight cardinal directions according to the orientation of the participant. In a similar way, they were then guided to the second, third, and fourth turning points and finally to the destination point. Experimenters indicated that the destination was reached after the participants had arrived at the destination point.

The force display was always "on" during the navigation. If the participant made a wrong turn (and was about to begin walking the wrong route), the experimenter changed the direction of the haptic stimuli manually to return the participant to the predefined route and sent them via the remote computer. The same haptic stimuli for the eight cardinal directions were used for the revision. Nevertheless, if this correction failed or the participants did not notice the stimulus change, the experimenter walking behind intervened by touching their backs, giving verbal information about the correct turn and taking note of any incorrect actions. Note that the participants were made aware of a wrong turn in an interruption but not in a revision. The participants had to go back one block to return to the correct path when there was an interruption.

The turn-by-turn navigation task consisted of walking predefined routes in the human-size maze under two auditory information conditions (audio masked and audio unmasked). Under the audio-masked condition, all the audio information was masked by noisecancelling headphones with white noise. Under the audio-unmasked condition, the participants were able to hear ambient sounds, but no artificial sounds were presented. We expected that people with visual impairments would utilize ambient audio information to identify obstacles or walls in front of them. Since the foam panels along the route were 900 $\mathrm{mm}$ high, the audio cues would be different from those provided when walking along an ordinary hallway. However, the wooden wall forming part of the building was over five meters high, and it provided a clear acoustic echo that assisted localization. Each participant completed one trial per one condition. All predefined routes were determined so that the departure and destination points were incongruent, and there were four turns. The routes were different for the two conditions and were randomly selected to reduce the learning effect, for example to prevent the distance to a turn being remembered or guessed. The 
participants were instructed to walk as fast and as accurately as possible. All participants were invited to complete our two-item questionnaire and to comment freely about what they felt during the task after the experiment. The statements were presented in a different randomized order for each participant. Each statement was rated by the participants on a seven-point Likert scale, with -3 meaning 'totally disagree' and +3 'totally agree'. The questions were:

Q1. The guidance was easy to understand.

Q2. I expect it would be useful in disaster situations.

Our intention with Q1 was to gain some insight into the usability of the force display in the experiment for users with visual impairments. The aim with Q2 was to gain some insight into the feasibility of using it during disasters, such as in heavy smoke (visual cues unavailable) or when there is a loud siren noise (audio cues unavailable). In Q2, the experimenter explained that a disaster meant a typical situation that lacked some audiovisual information, and which required quick and safe directional navigation. These questions focused on obtaining a subjective rating of the understandability and reliability of the force display and the navigation system.

\subsubsection{Results}

The experimental results show that our proposed system successfully enabled the participants to follow predefined routes. Twenty-one of twenty-three participants (i.e., 91\%) successfully walked from the entry point to the endpoint with four turns under both conditions. Figure 6 shows examples of walking trajectories. Note that trials revised by the experimenter were also counted as successes, whereas trials interrupted by the experimenter were counted as failures. The same two participants failed to complete the tasks under both conditions. The experimenter intervened twice under the audio-masked condition, and three times under the audio unmasked condition. No other participants required intervention during the navigation task. Specifically, one of those two participants always seemed to judge left stimuli as right stimuli, and the other interpreted right stimuli as left stimuli. This tendency appeared to prevent them from returning to the predefined route even if the stimuli were changed manually. Seven participants under the audio-masked condition and six under the audio- unmasked condition failed to perceive the force sensation indicating a turn. Two of them could not recover the route at all. The other participants were able to recover the route when the force display was driven by the experimenter. Under the audio-masked condition, five participants made five mistakes. Under the audio- unmasked condition, four made five mistakes. The average time required to recover from an incorrect turn was about five seconds, and the longest time was about ten seconds.

Almost all the participants rated both statements highly. The medians of the questionnaire results were +2 and +2 . The quartile ranges were 1 and 1 . No outliers were observed. Moreover, we analyzed the questionnaire results very conservatively by considering only the high scores $(+2$ or +3$)$ for each statement as indicating the usefulness; in other words, the seven-point questionnaire responses were converted into a binary response ('high score' or 'not high score'). The scientific motivation for this is that we wanted to perform an analysis that only took account of people who felt strongly about the usefulness of this force-based navigation. By chance alone, the probability of a high score is $2 / 7$. The ratings for the two 
statements have frequencies that are much higher than would be expected by chance $(21$ and 18 respectively out of 23 , with corresponding $p<.0001$ and .0001 , using the binomial distribution).

Some participants commented after performing the task that they felt the device to be very useful and made it easy to comprehend the direction. A negative comment was that it was hard to keep the force display horizontal and to maintain the direction indicated by the display for a long period of time. Another negative comment from one participant was that his hand felt numb because of the vibration and weight of the force display.
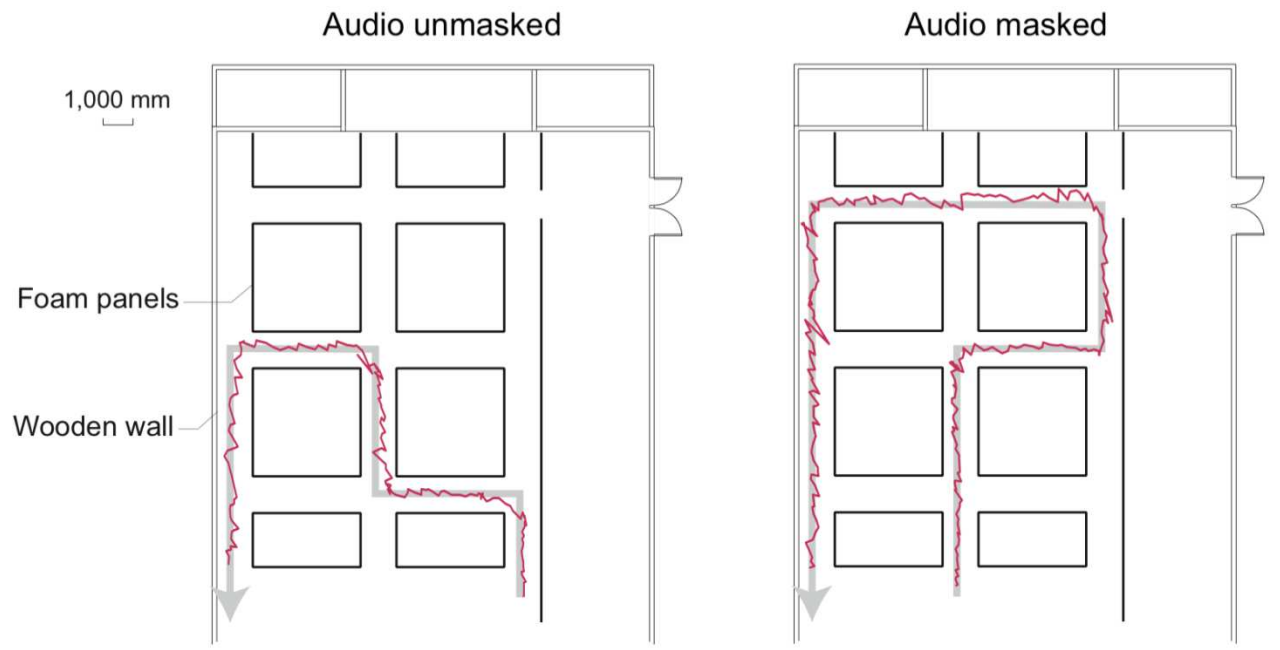

Fig. 6. Examples of a visually impaired participant's walking trajectories. Even if auditory information was masked, they could follow predefined routes

\section{Discussion and design implications}

The results provide clear evidence of the usefulness of force-based navigation for pedestrians with visual impairments. Some participants collided with the walls because the developed navigation system had no function for the recovery to a straight path from a meandering walk. Due to the lack of such a function, the participants' direction tended to diverge from the center line after they had turned a corner. To avoid such collisions, haptic signals for mid-course corrections are required. There is a trade-off between increasing the number of haptic signals and intuitive comprehension (or mental load), which is a version of the haptic icon problem (Enriquez and MacLean 2008). Nonetheless, this should be considered in future implementations. Our proposed system would also benefit from information displays for other sensory modalities. For instance, adding an audio navigation aid would make it relatively easily to provide complex and semantically rich information, such as categories of landmarks and street names. This semantic richness and complexity is very difficult to achieve with just a force display. Ross and Blasch pointed out that the combination of speech (auditory) and tapping (tactile) information would be useful as orientation aids (Ross and Blasch 2000). Future work will include investigating the effect of such additional meaningful audio information such as street names, and landmarks. 
Our haptic navigation system can easily be expanded to support sighted pedestrians. In particular, travellers with a different mother tongue visiting an area would reap significant benefits because haptic instruction is nonverbal. The proposed system would also enable users with reading disabilities to travel independently. Iconic symbols such as left or right arrows would be sufficiently clear and accurate to indicate directions. However, haptic cues not only indicate direction (at the sensory phase) but also make bodies move directly (at the motor phase), which requires less response time. Therefore, haptic cues would be more suitable for navigation applications. Future work will include the integration of satellite navigation instruction, which would expand the available area of our technique.

Manually changing the force vector assisted participants who made a wrong turn to return to the original route. Since the haptic stimuli were identical for the automatic and manual operations, one of the main reasons manual intervention was required was the incomplete algorithm for changing the direction of the force vector. The algorithm depended on the accuracy and reliability of the local position sensors (infrared sensors placed at the maze corners). The detection onset for the local position sensors and turning a corner varied as the walking pace changed.

Our experimental setup has some limitations with regard to generalizing the findings for all areas of pedestrian navigation since we only examined turn-by-turn navigation task in orthogonal-crossed route. Since most streets and avenues, with the exception of certain cities such as Kyoto, are not laid out in a grid, a navigation task in relation to complicated routes must be the next step. In addition, we have to clarify whether the same angular resolution (i.e., the eight cardinal directions) is sufficient for arbitrary routes, in particular when avoiding obstacles. Pielot et al. have reported deficiencies in the human perception of body orientation when small angles are involved (Pielot et al. 2008). Our previous study also showed that there is a systematic error with respect to the human perception of force orientation among people with visual impairments when they are not moving, which is less than 15 degrees when the force vector is provided in the eight cardinal directions (Amemiya 2009). A dynamic exploration of the force vector would be useful when walking complicated routes, which is similar to the guidance a human or a guide dog might provide and depends on the active or passive perception of haptic feedback while walking. Therefore, the systematic error could be minimized with a closed feedback loop, which could lead to precise routing. However, debate continues about the best way to understand the user's orientation for dynamic exploration, and about the most suitable part of the body to which to attach the orientation sensors. Moreover, we must consider whether the force display should be held in the hand, carried in a bag, or worn.

In the experiment, the force display always generated force during navigation, because in a pilot study we received feedback that the absence of stimuli made the participants anxious. However, it is well known that continued vibration tends to cause perceptual fatigue (i.e., adaptation) if it is presented for too long, as with all other sensations (Coren et al. 2003). Nevertheless, there were no differences in the number of collisions with the walls between the L-shaped walking paths of the same trial: one is from the entry point to the second turn, the other is from the second to the last turn to the exit point. The average numbers of collisions were 0.10 and 0.12 (audio unmasked condition), and 0.07 and 0.09 (audio masked), respectively. The average lengths of the former and latter paths were 11.2 meters and 13.2 meters (audio unmasked), and 9.4 meters and 14.2 meters (audio masked), respectively. In addition, all but one of the participants reported that they did not feel any subjective perceptual difference between the start and the end as mentioned above. We conjecture that 
the effect of vibrotactile adaptation may not appear during short-term navigation, since the asymmetrically oscillating stimuli involve not only cutaneous but also proprioceptive sensations. The muscle spindles or the Golgi tendon organs, which are receptors generating the proprioceptive sensation, are slowly adapting units, while the Pacinian corpuscles, receptors that detect skin vibration, are rapidly adapting units.

Many of the participants made similar positive comments. The questionnaire rating clearly revealed high confidence levels and high expectations. Their medians and quartiles confirmed that the force sensation was well perceived. Responses to Q1 indicated that the participants were aware of the direction information and found the information provided by our system to be very intuitive. Those to Q2 confirmed that many of the participants realized the importance of force feedback in emergency navigation. The participants also commented on the quietness of the force display. They commented that the noise level of the force display would be acceptable in daily life, such as in public spaces. People with visual impairments sometimes use the information provided by acoustic echoes to gain awareness of the environment. The proposed device was so quiet that this information could still be used.

It is crucial to miniaturize and lighten the force display so that it can be carried more easily. However, the amplitude of the kinesthetic stimuli, which should be large enough to be perceived, is proportional to the mass of the weight and the amplitude of acceleration. The trade-off between the mass of the weight and strength of perception limits the amount by which the weight can be reduced. However, the size of the force display could be reduced by using other mechanisms to generate similar asymmetric oscillation, such as linear actuators. We speculate that a miniaturized version of the force display could be embedded in canes for people with visual impairment. It is true that some people feel that no device should be attached to the cane, but only a relatively small amount of retraining would be needed.

\section{Related work}

Recent research has addressed a range of issues concerning pedestrian navigation aid. Pedestrian navigation systems on mobile devices, such as mobile phones with a satellite positioning function, that employ different sensory channels (i.e., visual and/or audio channels) are being increasingly developed. Since people with visual impairments cannot use vision-based navigation aids, many handheld auditory-feedback devices for people with visual impairments have been developed, such as Talking Signs (Crandall et al. 1999) or similar acoustic information output devices (Loomis et al. 2005). Although such audio interfaces help users move in the right direction by providing sound cues, they can be problematic when they conflict with other sounds or speech around the users, making it difficult for them to distinguish and interpret the sounds generated by the system (Wilson et al. 2007). In addition, pedestrians with visual impairments often rely on information contained within the ambient sounds for navigation purposes. Wearing headphones prevents them from hearing these ambient sounds thereby making navigation less safe. Furthermore, auditory information cannot be used in noisy situations, such as on busy city streets.

Tactile interaction may help overcome such navigation issues for people with visual impairments. It has been reported that tactile interaction can effectively assist pedestrians with visual impairments when crossing the street (Ross and Blasch 2000). As tactile-based 
navigational aids, vibrotactile stimulation systems that use several vibrators in the shape of a cap (Cassinelli et al. 2006), rings (Amemiya et al. 2004), a vest (Erp et al. 2005), belt (Tan et al. 2003; Heuten et al. 2008), and glove (Zelek et al. 2003) have been proposed. Unfortunately, these tactile approaches require that users learn how to convert stimuli to information. This is not intuitive and requires training since the tactile stimuli employed are basically non-directional.

\section{Acknowledgements}

We thank Dr. Ichiro Kawabuchi for his technical assistance. We also thank Hisashi Sugiyama, the staff of Kyoto City Fire Department, and the staff of the Kyoto Prefectural School for the Visually Impaired for their kind cooperation. This study was supported by Nippon Telegraph and Telephone Corporation and was also partially supported by the sponsorship of the Fire Defence Agency, Japan.

\section{References}

Amemiya, T.; Ando, H. \& T. Maeda T. (2005). Virtual force display: Direction guidance using asymmetric acceleration via periodic translational motion, Proceedings of World Haptics Conference, IEEE Computer Society, pp. 619-622.

Amemiya, T.; Ando, H. \& T. Maeda T. (2008). Lead-Me interface for pulling sensation in hand-held devices, ACM Transactions on Applied Perception, Vol. 5, No. 3, pp. 1-17.

Amemiya, T. \& Maeda, T. (2008). Asymmetric oscillation distorts the perceived heaviness of handheld objects, IEEE Transactions on Haptics, Vol. 1, No. 1, pp. 9-18.

Amemiya, T. \& Maeda, T. (2009). Directional force sensation by asymmetric oscillation from a double-layer slider-crank mechanism, Journal Computing Information Science in Engineering, Vol. 9, No. 1, 011001.

Amemiya, T.; Maeda, T. \& Ando, H. (2009). Location-free Haptic Interaction for Large-Area Social Applications, Personal and Ubiquitous Computing, Vol. 13, No. 5, pp. 379-386, Springer.

Amemiya, T. \& Sugiyama, H. (2009). Haptic Handheld Wayfinder with Pseudo-Attraction Force for Pedestrians with Visual Impairments, Proceedings of 11th ACM Conference on Computers and Accessibility (ASSETS 2009), Pittsburgh, PA, pp. 107-114.

Amemiya, T. \& Sugiyama, H. (2010). Orienting Kinesthetically: A Haptic Handheld Wayfinder for People with Visual Impairments, ACM Transactions on Accessible Computing, Vol. 3, No. 2, pp. 1-23.

Amemiya, T.; Yamashita, J.; Hirota, K. \& Hirose, M. (2004). Virtual Leading Blocks for the Deaf-Blind: A Real-Time Way-Finder by Verbal-Nonverbal Hybrid Interface and High-Density RFID Tag Space, In Proc. of IEEE Virtual Reality Conference 2004 (VR 2004), pp. 165-172.

Bradley, A. \& Dunlop, D. (2005). An experimental investigation into wayfinding directions for visually impaired people, Personal Ubiquitous Computing, Vol. 9, No. 6, pp. 395403.

Cassinelli, A.; Reynolds, C. \& Ishikawa, M. (2006). Augmenting spatial awareness with haptic radar. In Proc. International Conference on Wearable Computing. IEEE Computer Society, pp. 61-64. 
Coren, S.; Ward, L. M. \& Enns, J. T. (2003). Sensation and Perception. John Wiley and Sons, Inc.

Crandall, W.; Brabyn, J.; Bentzen, B. \& Myers, L. (1999). Remote infrared signage evaluation for transit stations and intersections. Journal of Rehabilitation Research and Development Vol. 36, pp. 341-355.

Enriquez, M. \& MacLean, K. (2008). The role of choice in longitudinal recall of meaningful tactile signals. In Proc. of 16th IEEE Symposium on Haptic interfaces for virtual environment and teleoperator systems. pp. 49-56.

Erp, J. B. F. V.; Veen, H. A. H. C. V.; Jansen, C. \& Dobbins, T. (2005). Waypoint navigation with a vibrotactile waist belt. ACM Transactions on Applied Perception, Vol. 2, No. 2, pp. 106-117.

Foulke, E. (1996). The roles of perception and cognition in controlling the mobility task. International Symposium on Orientation and Mobility.

Golledge, R. G. (1992). Place recognition and wayfinding: making sense of space. Geoforum Vol. 23, No. 2, pp. 199-214.

Gurocak, H.; Jayaram, S.; Parrish, B. \& Jayaram U. (2003). Weight sensation in virtual environments using a haptic device with air jets, Journal of Computing and Information Science in Engineering, Vol. 3, No. 2. ASME, pp. 130-135.

Hayward, V. (2008). A Brief Taxonomy of Tactile Illusions and Demonstrations That Can Be Done In a Hardware Store, Brain Research Bulletin, Vol. 75, pp. 742-752.

Heuten, W.; Henze, N.; Boll, S. \& Pielot, M. (2008). Tactile wayfinder: a non-visual support system for wayfinding. In NordiCHI. ACM International Conference Proceeding Series, Vol. 358. ACM Press, pp. 172-181.

Hirose, M.; Hirota, K.; Ogi, T.; Yano, H.; Kakehi, N.; Saito, M. \& Nakashige, M. (2001). HapticGEAR: The Development of a Wearable Force Display System for Immersive Projection Displays, Proceedings of Virtual Reality 2001 Conference, pp. 123-130.

Hoshi ,T.; Takahashi, M.; Iwamoto, T. \& Shinoda, H. (2010). Noncontact Tactile Display Based on Radiation Pressure of Airborne Ultrasound, IEEE Transactions on Haptics, Vol. 3, No .3, pp. 155-165.

Loomis, J.; Marston, J.; Golledge, R. \& Klatzky, R. (2005). Personal guidance system for people with visual impairment: A comparison of spatial displays for route guidance. Journal of Visual Impairment and Blindness Vol. 8, No. 5, pp. 61-64.

Massie, T. \& Salisbury, J. K. (1994). The phantom haptic interface: A device for probing virtual objects, Proceedings of the ASME Winter Annual Meeting, Symposium on Haptic Interfaces for Virtual Environment and Teleoperator Systems, Vol. 55-1, pp. 295-300.

Nakamura, N. \& Fukui, Y. (2007). Development of Fingertip Type Non-grounding Force Feedback Display, Proceedings of World Haptics Conference 2007, pp. 582-583.

Pielot, M., Henze, N., Heuten, W., \& Boll, S. (2008). Evaluation of continuous direction encoding with tactile belts. In Proc. the 3rd international workshop on Haptic and Audio Interaction Design, Springer, LNCS, pp. 1-10.

Richard, C. \& Cutkosky, M. (1997). Contact Force Perception with an Ungrounded Haptic Interface, Proceedings of the ASME Dynamic Systems and Control Division, pp. 181187.

Ross, D. \& Blasch, B. (2000). Wearable interfaces for orientation and wayfinding. In Proc. ACM Conference on Assistive Technologies. ACM Press, pp. 193-200. 
Suzuki, Y.; Kobayashi, M. \& Ishibashi, S. (2002). Design of force feedback utilizing air pressure toward untethered human interface, Proceedings of CHI '02 Extended Abstracts on Human Factors in Computing Systems. ACM Press, 2002, pp. 808-809.

Swindells, C.; Unden, A. \& Sang, T. (2003). TorqueBAR: an ngrounded haptic feedback device. Proceedings of the 5th international conference on multimodal interfaces. ACM Press, pp. 52-59.

Tan, H. Z.; Gray, R., Young, J. J. \& Traylor, R. (2003). A haptic back display for attentional and directional cueing. Haptics-e: The Electronic Journal of Haptics Research Vol. 3, No. 1.

Tanaka, Y.; Masataka, S.; Yuka, K.; Fukui, Y.; Yamashita, J. \& Nakamura, N. (2001). Mobile torque display and haptic characteristics of human palm. Proceedings of 11th international conference on augmented tele-existence, pp. 115-120.

Wilson, J.; Walker, B.; Lindsay, J.; Cambias, C. \& Dellaert, F. (2007). Swan: System for wearable audio navigation. In Proc. International Conference on Wearable Computing. IEEE Computer Society, pp. 91-98.

Yano, H.; Yoshie, M. \& Iwata, H. (2003). Development of a nongrounded haptic interface using the gyro effect, Proceedings of 11th international symposium on Haptic Interfaces for Virtual Environment and Teleoperator Systems. IEEE Computer Society, pp. 32-39.

Zelek, J. S.; Bromley, S.; Asmar, D. \& Thompson, D. (2003). A haptic glove as a tactile-vision sensory substitution for wayfinding. Journal of Visual Impairment and Blindness Vol. 97, No. 10, pp. 621-632. 


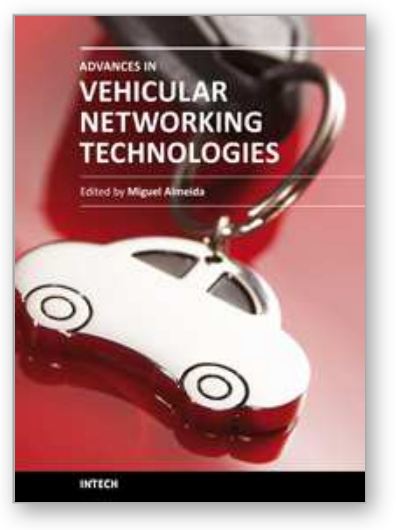

\author{
Advances in Vehicular Networking Technologies \\ Edited by Dr Miguel Almeida
}

ISBN 978-953-307-241-8

Hard cover, 432 pages

Publisher InTech

Published online 11, April, 2011

Published in print edition April, 2011

This book provides an insight on both the challenges and the technological solutions of several approaches, which allow connecting vehicles between each other and with the network. It underlines the trends on networking capabilities and their issues, further focusing on the MAC and Physical layer challenges. Ranging from the advances on radio access technologies to intelligent mechanisms deployed to enhance cooperative communications, cognitive radio and multiple antenna systems have been given particular highlight.

\title{
How to reference
}

In order to correctly reference this scholarly work, feel free to copy and paste the following:

Tomohiro Amemiya (2011). Kinesthetic Cues that Lead the Way, Advances in Vehicular Networking Technologies, Dr Miguel Almeida (Ed.), ISBN: 978-953-307-241-8, InTech, Available from:

http://www.intechopen.com/books/advances-in-vehicular-networking-technologies/kinesthetic-cues-that-leadthe-way

\section{INTECH}

open science | open minds

\section{InTech Europe}

University Campus STeP Ri

Slavka Krautzeka 83/A

51000 Rijeka, Croatia

Phone: +385 (51) 770447

Fax: +385 (51) 686166

www.intechopen.com

\section{InTech China}

Unit 405, Office Block, Hotel Equatorial Shanghai

No.65, Yan An Road (West), Shanghai, 200040, China

中国上海市延安西路65号上海国际贵都大饭店办公楼 405 单元

Phone: +86-21-62489820

Fax: $+86-21-62489821$ 
(C) 2011 The Author(s). Licensee IntechOpen. This chapter is distributed under the terms of the Creative Commons Attribution-NonCommercialShareAlike-3.0 License, which permits use, distribution and reproduction for non-commercial purposes, provided the original is properly cited and derivative works building on this content are distributed under the same license. 